\title{
ANALOGÍA MORFOLÓGICA Y MARCAJE FONOLÓGICO EN LOS VERBOS INCOATIVOS DEL CATALÁN*
}

\author{
Paula Cruselles \\ Universitat de València
}

\section{RESUMEN}

La analogía entre formas de un paradigma morfológico puede dar lugar a alomorfos marcados desde el punto de vista de la fonología. En este estudio nos centramos en el análisis del incremento incoativo -isc- [ísk] que se puede realizar en el catalán de Valencia con una sibilante palatal en los verbos incoativos de la tercera conjugación: por ej., servixc [servífk] 'sirvo'. A pesar de que el grupo consonántico [ $\left.\int \mathrm{k}\right]$ se considera marcado fonológicamente, esta forma palatalizada parece haber sustituido a la forma tradicional del catalán occidental con una sibilante alveolar-isc- [ísk], por analogía con formas del presente de indicativo como servix [servíf] 'sirve'. Los resultados del estudio indican que, a pesar de que las formas con -ixc-son muy frecuentes en la documentación escrita, no todos los hablantes presentan realmente una pronunciación palatal de la sibilante del grupo -isc- y que, en los casos en que se detecta la palatalización analógica en la pronunciación, la realización de la sibilante se encuentra a medio camino entre una sibilante alveolar y una sibilante palatal, en una especie de compromiso entre las restricciones fonológicas y las morfológicas.

PALABRAS ClAVE: analogía, incoativo, interfaz fonología-morfología, morfología natural, catalán.

\section{MORPHOLOGICAL ANALOGY AND PHONOLOGICAL MARKEDNESS \\ IN THE INCHOATIVE VERBS OF CATALAN}

\section{Abstract}

Analogy between forms of a morphological paradigm can give rise to phonologically marked allomorphs. In this study, we focus on the analysis of the inchoative form -isc- [ísk] in Valencian Catalan, which can be realized with a palatal sibilant in 3rd conjugation inchoative verbs: e.g., servixc [servífk] 'I suffer'. Although the consonantal cluster [ $\int \mathrm{k}$ ] is considered phonologically marked, this palatalized form, coined by analogy with other indicative present forms such as servix [servíf] 's/he suffers', seems to have replaced the traditional western Catalan form with an alveolar sibilant -isc- [ísk]. Our results indicate that, although the forms with -ixc-are very common in written documentation, not all speakers pronounce the sibilant segment as a palatal consonant. Additionally, the data show that, in cases where the analogical process affects the pronunciation, the realization of the sibilant is halfway between an alveolar sibilant and a palatal sibilant, in a kind of compromise between phonological and morphological conditions.

KEYWORDs: analogy, inchoative forms, phonology-morphology interface, natural morphology, Catalan. 


\section{INTRODUCCIÓN}

La analogía entre formas de un paradigma morfológico puede dar lugar a alomorfos marcados desde el punto de vista de la fonología. En este trabajo, presentamos diferentes modelos de realización de las formas del incremento incoativo verbal del catalán de Valencia, representado como -isc- en la ortografía actual (AVL 2006: 247), como un ejemplo que ilustra el conflicto entre la presión morfológica del paradigma y las restricciones fonológicas en contra de ciertas configuraciones segmentales. En valenciano se han documentado dos representaciones gráficas alternativas para los verbos de la tercera conjugación con incremento incoativo: por un lado, encontramos la forma verbal tradicional del catalán occidental, con el incremento -isc- con la grafía $<s>$, que representa la alveolar sorda [s], como en servisca [servíska] (1a), y, por otro lado, aparece una forma alternativa con la grafía $\langle\mathrm{x}\rangle,-i x c$-, que normalmente representa la alveolopalatal sorda $[\mathrm{S}]$, como en servixca [servílka] (1b).

a. No pense que la clau servisca 'No pienso que la llave sirva'

b. No pense que la clau servixca 'No pienso que la llave sirva'

La existencia de esta doble forma para un mismo significado revela la pugna entre dos presiones latentes: por una parte, en catalán no se permite una secuencia con una sibilante palatal en la coda seguida de otra consonante, en la misma coda o en el ataque de la sílaba siguiente (Wheeler 1987: 86); es decir, la pronunciación [servífka] atenta contra las restricciones segmentales de carácter secuencial de la lengua, ya que presenta el fonema alveolopalatal sordo / $/$ / en la coda seguido de la consonante $/ \mathrm{k} /$ en posición inicial de sílaba. Esta condición fonológica favorecería el mantenimiento de la forma tradicional con alveolar sorda /s/: [servíska]. Por otra parte, las restricciones de tipo morfológico empujan el sistema en la dirección contraria: la forma servisca transgrede el principio de uniformidad morfológica (v. Wheeler 1993 y las referencias que se citan en esta obra), puesto que el mantenimiento de -isc-implica la existencia de más de una pronunciación -de más de una forma- para el incremento incoativo en el modo indicativo: una con una consonante alveolar en el incremento -isc- [ísk]: por ej., servisc [servísk] 'sirvo', y otra con consonante palatal en el incremento -ix-: por ej., servix [servíf] 'sirve'. Para evitar esta irregularidad en el paradigma, se tendría que imponer o bien la despalatalización de las formas con -ix-, o bien la extensión de la palatalización a formas como servixca. La documentación gráfica parece indicar que la solución mayoritaria en la lengua es la segunda ${ }^{1}$.

* Esta investigación ha sido financiada por el programa predoctoral VALi+D (ACIF/2016/340) de la Generalitat Valenciana y forma parte del proyecto FFI2016-76245-C3-3-P, financiado por la Agencia Estatal de Investigación y por el FEDER (http://www.ub.edu/GEVAD), y del grupo GIUV2013-137, de la Universitat de València. Agradezco los comentarios de los asistentes al XV Encuentro de Morfólogos Españoles: Morfología y Semántica de La Laguna, así como las 
La duplicidad de formas ha generado un gran debate entre algunos lingüistas sobre cuáles son las formas ortográficas que debe adoptar la normativa en el ámbito de Valencia. Entre los precedentes más relevantes de la forma clásica con la alveolar sorda $<$ s $>$ (-isca), que es la adoptada finalmente por la autoridad normativa (AVL 2006: 247), se encuentran Sanchis Guarner (1950) o Pérez Saldanya (1998). Otros lingüistas, en cambio, como Saragossà (2005) o Saborit (2009), han defendido que la normativa tendría que reflejar la realidad de la pronunciación de los hablantes, que de acuerdo con estos autores corresponde a la realización palatal de la sibilante; por tanto, proponen que la grafía que tendría que adoptarse en la escritura corresponda a la alveolopalatal sorda $/ \mathrm{f} /:<\mathrm{x}\rangle$.

Teniendo en cuenta el predominio de la forma servixca en la documentación escrita a partir del siglo xv, se podría pensar que, en la batalla entre restricciones fonológicas y regularidad morfológica, han ganado los principios que priman la regularidad en el paradigma por encima de las restricciones segmentales de carácter secuencial. La pregunta metodológica que nos planteamos en este estudio es si la grafía -ixc-encontrada en la documentación trasciende a la escritura y realmente deriva de una pronunciación alveolopalatal de la sibilante o si, por el contrario, se trata solo de una analogía puramente gráfica, sin un correlato en la pronunciación. En el primer caso, esperaríamos encontrar en el ámbito oral una realización como $\left[\int\right]$ de la sibilante, de manera que habría una correspondencia exacta entre el sonido y la grafía $<x>$ que representaba tradicionalmente a la consonante sibilante en estas formas; en el segundo, a pesar de que los usuarios puedan tender a representar la sibilante como $<\mathrm{x}>$, se pronunciaría como una $[\mathrm{s}]$.

El objetivo de este estudio es, pues, comprobar hasta qué punto los valencianohablantes realizan el incremento incoativo -isc- con alveolopalatal sorda [ $\int$ ], hecho que podría justificar la presencia de la grafía $\langle\mathrm{x}\rangle$ en este incremento. Para conseguir ahondar en el tema, en primer lugar, comentaremos el origen de los incrementos incoativos y su evolución en el sistema catalán (sección 2). En segundo lugar, expondremos la metodología del análisis experimental diseñado para estudiar el tipo de sibilante que realizan en el incremento palatal los sujetos valencianohablantes seleccionados (sección 3). A continuación, comentaremos los resultados extraídos del estudio estadístico, que indican las características acústicas de las diferen-

sugerencias de los dos revisores anónimos y también de Manuel Badal, Emili Casanova, Jesús Jiménez, Maria-Rosa Lloret y Manuel Pérez Saldanya.

${ }^{1}$ En las variedades que no permiten una palatal preconsonántica se puede considerar que existe una neutralización de punto de articulación entre $/ \mathrm{s} / \mathrm{y} / \mathrm{J} /$, que se realizarían siempre como [s]. Teniendo en cuenta, sin embargo, que $[\delta]$ puede aparecer en las codas finales del catalán, como en servix, Recasens (1993: 155) sugiere que no resulta pertinente postular un proceso de neutralización en estos casos. Conviene notar, por otra parte, que la tolerancia del valenciano hacia la presencia de $[S]$ delante de consonante en algunas formas verbales tiene el potencial de activar el contraste entre /s/ y / / / en posición de coda preconsonántica, como mostraría el par mínimo visc [vifk] 'vivo' vs. visc [visk] 'visco, liga'. 
tes sibilantes analizadas (sección 4). En la sección 5 presentaremos las conclusiones del estudio (sección 5).

\section{ORIGEN DEL INCREMENTO -IXC- Y EXTENSIÓN DEL CAMBIO}

El incremento -isc- proviene de los verbos incoativos del latín, como FLoRESCO, a los que añadía un matiz de cambio de estado (Pérez Saldanya 1998: 98). En latín tardío, el antiguo afijo incoativo pierde su significado original y en catalán se reinterpreta como marca de conjugación de una subclase de verbos de la III conjugación, como servir. Más específicamente, esta marca se encuentra en distribución complementaria con la vocal temática $i$ característica de los verbos de la tercera conjugación. Esto explica que el incremento solo aparezca en las formas verbales en las que los verbos de la tercera conjugación no adoptan la vocal temática $i$. En los textos catalanes medievales predomina el incremento incoativo -sc-con la vocal $e$ (-esc-) en los dos bloques dialectales: tanto en catalán oriental como en catalán occidental. Con todo, en algunos textos del catalán empiezan a aparecer variantes del incremento con la vocal $i(-i s c-)$ en las formas de las personas cuarta y quinta de subjuntivo, seguramente por efecto analógico con las personas cuarta y quinta del presente de indicativo (v. Pérez Saldanya 1998: 110). En estas personas del presente de indicativo la vocal $i$ es tónica y funciona como marca conjugacional, hecho que provocaría que el infijo (-esc-), que no aporta ningún contenido semántico, pierda su motivación morfológica y desaparezca en estas formas. Como consecuencia de la influencia de las formas cuarta y quinta del indicativo sobre las del subjuntivo, como se ilustra en (2), los verbos incoativos pasan a presentar alomorfía entre -esce -isc- en la forma del incremento en subjuntivo (Pérez Saldanya 1998: 109); esto es, se genera un problema de uniformidad en las terminaciones.

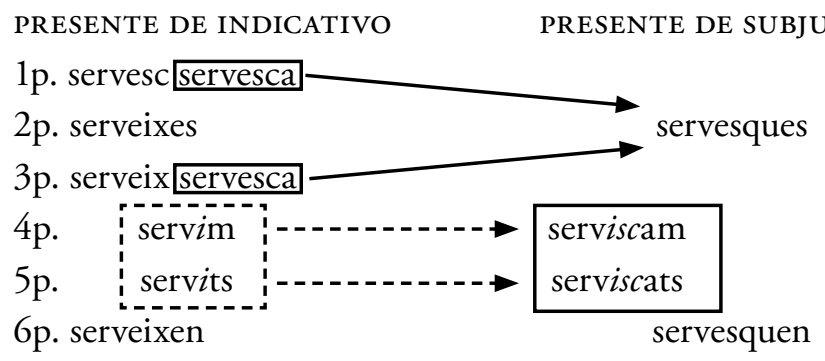

A partir del siglo XIV, en textos occidentales se observa que la forma -isc- de las personas cuarta y quinta del subjuntivo se extiende analógicamente a las otras formas verbales (Pérez Saldanya 1998: 110). En (3) se muestra un esquema orientativo de esta extensión. De esta forma, se elimina una de las fuentes de variación alomórfica de la conjugación, es decir, aumenta la uniformidad formal del sistema. 
1p. servesc

2p. serveixes

3p. serveix

4p. servim

5 p. servits

6p. serveixen

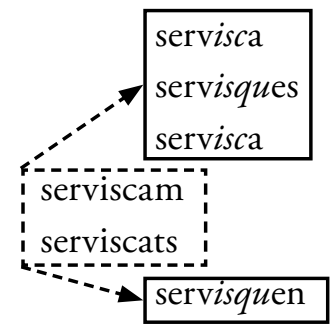

A partir de ese momento, la forma del incoativo con la vocal $i$ (-isc-) se convierte en una característica definitoria del catalán occidental, que lo diferencia del bloque oriental, en el que aparece la forma con la vocal $e$ (-esc-). En la mayor parte del bloque dialectal occidental, la pronunciación de la semivocal palatal [j] delante de la sibilante alveolopalatal sorda $/ \mathrm{S} /$, como en serveix [servéj $\int$ ], permite reducir el diptongo $e i$ en $i$. De acuerdo con Casanova (1993), esto explica, junto con la fuerza niveladora de la vocal $i$, el triunfo de las formas con la vocal $i$, que se extiende también a las restantes formas del indicativo (4). En el dialecto oriental del catalán, la semivocal palatal no se pronuncia: serveix [servéf]; por lo tanto, en este territorio la presión de la vocal $i$ es menor y se mantienen las formas con la vocal $e$.

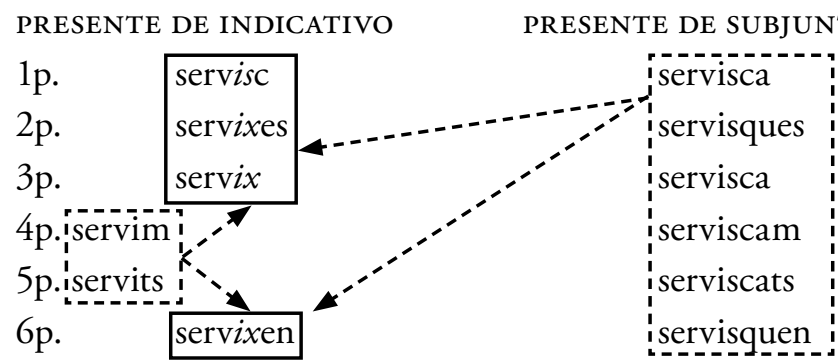

Con el cambio de (4) se reduce aún más la alomorfía, pero sigue habiendo formas que contienen un incremento incoativo alveolar, $-i s-$, al lado de otras formas con incremento incoativo palatal, $-i x$-. Posteriormente, en valenciano coloquial el segmento -isc-del presente de subjuntivo evoluciona a -ixc- (5), con una sibilante palatal preconsonántica, por analogía con las formas - $i x$ - del presente de indicativo. De esta manera, se consigue regularizar el paradigma y tener una sola forma con - $i x$ en todas las personas posibles (Saragossà 2005: 287; Saborit 2009: 72)².

${ }^{2}$ En un proceso independiente que no discutimos aquí, las formas cuarta y quinta del subjuntivo pierden el incremento incoativo por influencia de las formas paralelas del indicativo (v. Pérez Saldanya 1998: 110). 


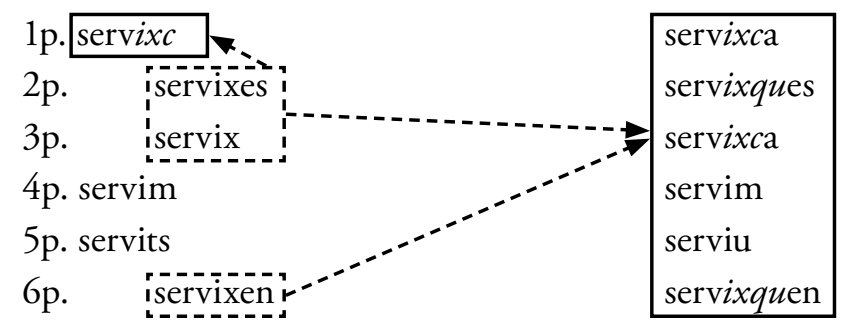

Regularizaciones según el patrón analógico de (5) son frecuentes en la documentación del catalán occidental desde aproximadamente el siglo xv, en que se documentan formas como (jo) servixca '(yo) sirva' o (jo) vixca '(yo) viva'. Estas grafías obedecerían en principio a una regularización morfológica. Con todo, la realización palatal de la sibilante también se puede haber visto favorecida por la influencia de la consonante velar posterior, como ha sucedido en otras formas no verbales como mosca ['mofka], fosc ['fofk], las cuales tienen una extensión reducida (Recasens 1996: 270, 2014: 257; Maria-Rosa Lloret 2002: 215; Segura-Llopes 2003:70; Beltran y Segura-Llopes 2017: 173) y, en pesca ['pe $k \mathrm{ka}$ ] (esta última forma está más extendida que las anteriores, seguramente también por la influencia de la forma relacionada morfológicamente con consonante palatal peix ['pejf]).

Sin embargo, la existencia de documentación abundante con la grafia $\langle\mathrm{x}\rangle$ no significa que necesariamente la secuencia -ixc-se pronunciara realmente con una consonante palatal [S]. Podría suceder, en efecto, que la analogía se limitara a las formas escritas y que la pronunciación siguiera respetando la restricción en contra de las consonantes palatales en posición final de sílaba preconsonántica definida por Wheeler (1987). Para aportar luz sobre esta cuestión, en este trabajo, a partir de una pequeña muestra de hablantes valencianos, analizaremos los datos acústicos de la consonante sibilante del incremento incoativo para comprobar si, efectivamente, la analogía se ha extendido a la pronunciación o si, por el contrario, opera exclusivamente en el ámbito de la ortografía.

\section{METODOLOGÍA}

Para este estudio se ha grabado a 3 mujeres valencianas de entre 20 y 24 años, procedentes de Albocàsser, Castellón (MC); de Riba-roja, Valencia (MB), y de Crevillent, Alicante (SM). La selección de estas participantes responde a la voluntad de estudiar una representante prototípica de cada uno de los grados de distinción entre /s/ vs. / / que se han establecido para el catalán de Valencia en estudios precedentes (v. Cruselles, en preparación). Esta gradación presenta tres estadios: la distinción nula, esto es, la neutralización entre las dos consonantes, representada por el sujeto $\mathrm{MC}$ de Albocàsser; la distinción mínima, ejemplificada por la informante $\mathrm{MB}$ de Riba-roja, y la oposición robusta, ilustrada con la informante SM de Crevillent.

Todas las informantes tienen padres valencianohablantes que proceden de la misma comarca en la que se sitúa la localidad de las informantes. Las grabaciones se 
han desarrollado en entornos silenciosos con un micrófono cardioide y de condensador (AKG C520L) y una grabadora digital (Zoom H4), con una frecuencia de muestreo de $48 \mathrm{kHz}$ y una calidad de 32 bits. Las informantes han accedido de forma voluntaria a realizar las grabaciones y no han recibido compensación económica.

Para obtener los datos acústicos de la sibilante palatal del incremento -isc-, hemos usado un corpus de nueve oraciones, que las informantes tenían que traducir del castellano al catalán (v. tabla 1). Se han escogido estos ejemplos para que, en la versión traducida al catalán, obtengamos 2 tipos de oraciones: las que contienen el elemento objeto de estudio: /-isk-/ (a partir de ahora: contexto aclarisca) y las que funcionan de contexto de control. En este último grupo de oraciones, tenemos, por un lado, un contexto en que la sibilante sorda final de palabra aparece precedida de la vocal $i$ y seguida, en la palabra siguiente, de una consonante oclusiva sorda velar /-ís\#\#k-l, englobadas a partir de ahora bajo el nombre de contexto París, y, por otro, un entorno en que la sibilante alveolopalatal sorda final de palabra aparece precedida de la vocal $i$ y seguida, en la palabra siguiente, también de una consonante oclusiva sorda velar /-ij\#\#k-/ (a partir de ahora: contexto aclarix). En los dos últimos contextos, para evitar un posible descenso de la frecuencia del CDG causado por la realización de una pausa entre palabras, se ha controlado que no existan pausas entre la sibilante sorda en final de palabra y la consonante oclusiva sorda velar situada en la palabra siguiente. De esta manera, con los dos grupos de control podremos evaluar si las sibilantes del contexto aclarisca analizado se realizan más cercanas a una alveolar sorda o a una alveolopalatal sorda. Las informantes han repetido diez veces las oraciones, de las cuales se han analizado siete repeticiones. Como norma, se han descartado siempre la primera y la última repetición para evitar problemas en la articulación: la primera repetición, para evitar posibles equivocaciones por desconocimiento de la palabra, y la última, para evitar la influencia de la bajada tonal típica de los últimos elementos de una enumeración. La antepenúltima repetición se grabó como comodín por si en la segmentación y el etiquetaje se descubría una anomalía en alguna de las siete repeticiones restantes (por ejemplo, una pausa en la frontera entre palabras).

\begin{tabular}{|c|c|c|}
\hline \multicolumn{3}{|c|}{$\begin{array}{l}\text { TABLA 1. ORACIONES MARCO PARA EXTRAER LOS DATOS ACÚSTICOS } \\
\text { DE LA SIBILANTE DEL INCREMENTO INCOATIVO -ISC- }\end{array}$} \\
\hline Contexto & Oraciones en Castellano & ORACIONES ESPERADAS EN CATALÁN \\
\hline \multirow{3}{*}{$\begin{array}{c}\text { /-isc-I } \\
\text { (aclarisca) }\end{array}$} & Quiere que lo aclare de día & Vol que ho aclarisca de dia \\
\hline & Quiere que se reúna de día & Vol que es reunisca de dia \\
\hline & No quiere que sufra de día & No vol que patisca de dia \\
\hline \multirow{3}{*}{$\begin{array}{l}\text { /-ís\#\#k-/ } \\
(\text { París })\end{array}$} & Trabaja en París cada día & Treballa a París cada dia \\
\hline & María bebe anís cada día & Maria beu anís cada dia \\
\hline & Suma un nuevo matiz cada día & Suma un nou mat $i$ s cada dia \\
\hline \multirow{3}{*}{ 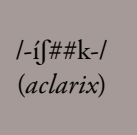 } & Me lo aclara cada día & M'ho aclarix cada dia \\
\hline & María lo sufre cada día & Maria ho patix cada dia \\
\hline & María se reúne cada día & Maria es reun $i x$ cada dia \\
\hline
\end{tabular}


El análisis acústico de las consonantes sibilantes sordas se ha realizado con el programa Praat (Boersma y Weenink, 2014). La segmentación de las consonantes y el etiquetaje se ha realizado de manera manual a partir de la información que proporciona el oscilograma y el espectrograma. La señal se ha filtrado por encima de los $11000 \mathrm{~Hz}$, porque esta región no se considera relevante para el estudio de las sibilantes fricativas sordas, y también por debajo de los $1000 \mathrm{~Hz}$, para evitar la influencia de los rastros de una posible sonorización parcial por la influencia de la vocal precedente. Para caracterizar las consonantes, se ha utilizado el primer momento espectral, el centro de gravedad (a partir de ahora, CDG), que indica la frecuencia mediana de concentración de la energía en el espectro de un sonido. Esta propiedad espectral se relaciona con el punto de articulación de las sibilantes sordas: un valor del CDG más bajo indica una realización más posterior, mientras que un valor más elevado apunta a una producción más anterior (Jongman, Wayland y Wong 2000). Para extraer el centro de gravedad de las sibilantes estudiadas, se ha utilizado un script de Praat. Con este script se extrae el primer momento espectral a partir de un período de $20 \mathrm{~ms}$ situado en el centro de la fricción de la consonante, con el objeto de evitar la contaminación de las transiciones hacia los sonidos contiguos.

A partir de los valores de CDG obtenidos se han realizado tres pruebas ANOVA (una para cada sujeto; v. tabla 2) de un factor con el programa SPSS versión 24 (IBM Corp., 2016). En cada una de estas pruebas, se ha tomado como variable dependiente los valores individuales de CDG de cada realización de las consonantes sibilantes en los diferentes contextos estudiados y como variable independiente los tres contextos en que estas consonantes aparecen. Es decir, nuestro objetivo es saber si existen diferencias en los valores del CDG de las consonantes (variable dependiente) que se puedan deducir de los tres contextos en que aparecen (variable independiente). Como es habitual en las ciencias experimentales, el nivel de significación se sitúa en $\mathrm{p}=0,05$. En caso de que se observen diferencias en el CDG en función del contexto, se ha aplicado la prueba post-hoc de Scheffé, que nos permite conocer si se agrupan y cómo se agrupan los tres contextos y el valor de probabilidad de sus posibles agrupaciones; la escala de probabilidad va de 0 a 1 : cuanto menor sea el valor, menor será el grado de cohesión del grupo, y cuanto mayor sea el valor, mayor será la consistencia del grupo.

\begin{tabular}{|c|c|}
\hline VARIABLE INDEPENDIENTE: CONTEXTO & VARIABLE DEPENDIENTE \\
\hline 1. Contexto /-isk-/ (aclarisca) & \multirow{3}{*}{ CDG } \\
\hline 2. Contexto /-is\#\#k-/ (París) & \\
\hline 3. Contexto /-í $\{\# \# \mathrm{k}-/$ (aclarix) & \\
\hline
\end{tabular}




\section{RESULTADOS}

En este apartado analizaremos los resultados de los tres test ANOVA de un factor realizados para determinar las características acústicas de la sibilante del incremento /-isk-/ (contexto aclarisca) en relación con el entorno /-ís\#\#k-/ (contexto Paris) y el entorno /-íf\#\#k-/ (contexto aclarix) en las tres informantes seleccionadas.

\subsection{VARIEDAD CON DISTINCIÓN NULA}

Comenzamos el análisis con los resultados del test ANOVA de un factor usado para determinar la pronunciación de la sibilante del incremento -isca característica del sujeto $\mathrm{MC}$ de Albocàsser. Esta informante pertenece a una variedad de valenciano que, según la bibliografía, no presenta el fonema / $/$ / en su sistema fonológico porque se ha neutralizado con la consonante alveolar sorda /s/ (Colon 1970: 333; Recasens 2014: 245; Beltran y Segura-Llopes 2017: 171). De esta manera, en esta variedad las consonantes sibilantes del valenciano general caixa [kájfa] y caça [kása] confluyen en una consonante alveolar sorda: [kájsa] y [kása], respectivamente; una parte del contraste se puede mantener, de todas formas, por la presencia de la semivocal / j/ en [kájsa]. Este fenómeno se extiende y ya se documenta [s] en vez de $\left[\int\right]$ en otros puntos geográficos, como Alcoi, Ontinyent, Gandia, Alberic, València o Sagunt (Saborit 2009: 69) y también entre jóvenes de algunos pueblos de la comarca de l'Alcoià, el Comtat y la Vall d'Albaida (Beltran y Segura-Llopes 2017: 171)3.

Como los contextos de control, París y aclarix, también se pronuncian con neutralización de las sibilantes ([parís] y [aklarís]), nuestras expectativas respecto de la informante es que realice también el incremento -isca con alveolar sorda. El análisis del CDG revela, en efecto, que no existen diferencias en la realización de las sibilantes sordas de los tres contextos estudiados $([\mathrm{F}(2,60)=1,408, \mathrm{p}>0,252)$. $\mathrm{Si}$ se observa la tabla 3 , podemos comprobar que las sibilantes producidas por esta informante se sitúan en frecuencias altas, alrededor o por encima de los $6000 \mathrm{~Hz}$, que normalmente se asocian con las frecuencias en que se produce la sibilante alveolar sorda /s/. Este resultado confirma la hipótesis de la neutralización de la alveolar sorda /s/ del contexto París con la alveolopalatal sorda /g/ del contexto aclarix. Igualmente, se ajusta a las expectativas la realización de la sibilante en el incremento incoativo del contexto aclarisca, que no es diferente de los otros contextos y se sitúa, como muestra la media, en los valores propios de una sibilante alveolar. Para corro-

${ }^{3} \mathrm{Al}$ margen de la neutralización generalizada entre /s/y/s/ en algunas variedades del valenciano, ilustrada con la informante de Albocàsser, se ha documentado en otros dialectos una cierta tendencia despalatalizadora de / $\mathrm{f} /$ en algunas palabras como xarop [eisa'rop] 'jarabe', xifra ['sifra] 'cifra' o Xixona [si'sona] 'Jijona' (Recasens 2014: 245, Beltran y Segura-Llopes 2017: 174). 
borar los resultados, la prueba post-hoc de Scheffé ${ }^{4}$ nos demuestra que las sibilantes de la informante $\mathrm{MC}$ son muy similares y se pueden incluir en una misma agrupación con una significación relativamente elevada $(\mathrm{p}=0,270)$.

\begin{tabular}{|c|c|c|c|c|}
\hline \multicolumn{5}{|c|}{$\begin{array}{l}\text { TABLA 3. MEDIAS, DESVIACIÓN TÍPICA Y AGRUPACIONES (SEGÚN EL TEST POST-HOC } \\
\text { DE SCHEFFÉ REALIZADO A PARTIR DEL VALOR DE CENTRO DE GRAVEDAD) DE LAS } \\
\text { SIBILANTES EN LOS CONTEXTOS /-sk-/, /-îf\#\#k-/ Y /-ís\#\#k-/, CORRESPONDIENTE } \\
\text { A LOS DATOS DE LA INFORMANTE MC DE ALBOCÀSSER }\end{array}$} \\
\hline \multirow[t]{2}{*}{ Contexto } & \multirow[t]{2}{*}{ Casos } & \multirow[t]{2}{*}{ Media } & \multirow[t]{2}{*}{ Desviación tíPICA } & $\begin{array}{c}\text { Prueba POST-HOC DE SChefFÉ, } \\
\text { SUbCONJUNTOS PARA ALFA }=0,05\end{array}$ \\
\hline & & & & Grupo 1 \\
\hline aclarisca & 21 & 5.916 & 779 & 5.916 \\
\hline aclarix & 21 & 6.033 & 660 & 6.033 \\
\hline Paris & 21 & 6.303 & 853 & 6.303 \\
\hline \multicolumn{4}{|c|}{ Significación } & 0,270 \\
\hline
\end{tabular}

Como muestra el gráfico representado en la figura 1, las medias del centro de gravedad para los tres contextos apuntan a una articulación muy parecida. Es cierto que se advierte que las sibilantes del contexto París se realizan en unas frecuencias un poco más elevadas y, por tanto, con una articulación más adelantada que la de las sibilantes del contexto aclarisca y del contexto aclarix, que de acuerdo con el gráfico se producen en frecuencias casi equivalentes. Aunque este dato parezca apuntar a un cierto mantenimiento de la palatalidad de la consonante del contexto aclarix, la pequeña diferencia acústica observada no es estadísticamente relevante, como ha indicado el ANOVA realizado.

En definitiva, la neutralización de / / / de esta informante impide comprobar si existe analogía en el incremento o si, por el contrario, triunfa la restricción fonológica en contra del grupo [ $\mathrm{k} \mathrm{k}]$. Los datos referidos a esta hablante, sin embargo, nos sirven como un elemento de control para constatar una cierta tendencia de la consonante sibilante a realizarse un poco más retrasada en contacto con $/ \mathrm{k} /$ dentro de la palabra (contexto aclarisca).

\subsection{VARIEDAD CON DISTINCIÓN MÍNIMA}

A continuación, comentaremos los resultados del ANOVA de un factor aplicado a los datos del sujeto MB de Riba-roja. Este sujeto presenta una distinción mínima entre la alveolar sorda /s/ y la alveolopalatal sorda / $/$ en posición final de palabra, de aproximadamente $500 \mathrm{~Hz}$, que es la considerada prototípica del cata-

${ }^{4}$ Hemos realizado la prueba post-hoc de Scheffé con esta informante, pese a que no hay diferencia entre los contextos, solo para mostrar al lector que las diferentes realizaciones se organizaban en un mismo grupo. 


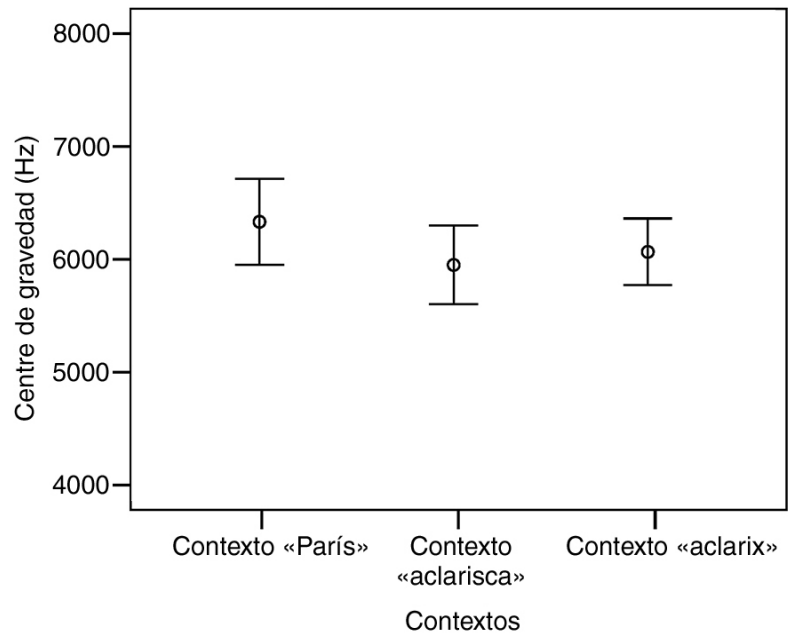

Figura 1. Gráfico de la media del CDG, con barras de error, de las sibilantes para la informante MC de Albocàsser en los contextos /-ís\#\#k-/ (París), /-sk-/ (aclarisca) y /-íj\#\#k-/ (aclarix).

lán de Valencia. De acuerdo con Recasens (2014) y con Cruselles et al. (2017), en valenciano la distancia entre las sibilantes es más reducida que en otros dialectos como el catalán oriental (Recasens 1986, 2014) o el mallorquín (Recasens y Espinosa 2006, 2007; Recasens 2014). Esta reducción de la distancia acústica entre las sibilantes en el catalán de Valencia se atribuye normalmente a la realización más anterior, es decir, más alveolar de la alveolopalatal sorda / // Recasens y Espinosa 2006, 2007; Saborit 2009; Recasens 2014). La realización más adelantada de / / / puede ilustrarse con los datos de esta hablante, que se sitúan alrededor de los $6000 \mathrm{~Hz}$, frecuencia que en otros dialectos mencionados del catalán suele corresponder a la sibilante alveolar sorda $/ s /$.

El estudio del centro de gravedad muestra que la realización de las sibilantes de la informante de Riba-roja es estadísticamente diferente en función de la variable 'contexto' $([\mathrm{F}(2,60)=8,129, \mathrm{p}=0,001])$. Como se puede comprobar en la tabla 4, las medias de los valores del CDG de las sibilantes de los contextos París y aclarisca están muy cercanas entre sí, superando en ambos casos los $6500 \mathrm{~Hz}$; en cambio, la media de las sibilantes del contexto aclarix se sitúa ligeramente por encima de los $6000 \mathrm{~Hz}$. El resultado para la distinción /s/ - / / / entre los contextos París y aclarix se sitúa en los márgenes esperables, ya que, como se ha comentado, la distancia entre la alveolar sorda /s/ y la alveolopalatal sorda / $/$ / suele ser reducida en valenciano, aunque es suficientemente notoria para ser estadísticamente distinta. Con la prueba post-hoc de Scheffé (v. tabla 4) comprobamos que las sibilantes realizadas por la informante $\mathrm{MB}$ se distribuyen en dos grupos con una significación altamente consistente ( $\mathrm{p}=1)$. Por un lado, encontramos la sibilante del contexto aclarix, con el valor medio de CDG más bajo $(6043 \mathrm{~Hz})$; mientras que, por otro lado, se observa 
un segundo grupo formado por las sibilantes del contexto Paris y del contexto aclarisca, con valores más elevados: $6572 \mathrm{~Hz}$ y $6577 \mathrm{~Hz}$, respectivamente 5 .

\begin{tabular}{|c|c|c|c|c|c|}
\hline $\begin{array}{r}\text { TABLA } 4 \\
\text { DE SCH } \\
\text { SIBII }\end{array}$ & $\begin{array}{l}\text { 1EDIAS } \\
\text { FÉ REA } \\
\text { NTES E }\end{array}$ & $\begin{array}{l}\text { ESVIAC } \\
\text { ZADO A } \\
\text { OS COI } \\
\text { OS DAT }\end{array}$ & $\begin{array}{l}\text { N TÍPICA Y AGRUP } \\
\text { RTIR DEL VALOR } \\
\text { EXTOS /-íj\#\#k-l, /-ís } \\
S \text { LA INFORMANT }\end{array}$ & $\begin{array}{l}\text { NES (SEC } \\
\text { ENTRO I } \\
\text { Y /-sk-I, C } \\
\text { DE RIBA }\end{array}$ & $\begin{array}{l}\text { OST-HOC } \\
\text { ENTE DE LAS }\end{array}$ \\
\hline \multirow[t]{2}{*}{ Contexto } & \multirow[t]{2}{*}{ Casos } & \multirow[t]{2}{*}{ Media } & \multirow[t]{2}{*}{ DESVIACIÓN TÍPICA } & \multicolumn{2}{|c|}{$\begin{array}{c}\text { PRUEBA POST-HOC DE SChefFÉ, } \\
\text { SUBCONJUNTOS PARA ALFA }=0,05\end{array}$} \\
\hline & & & & 1 & 2 \\
\hline aclarix & 21 & 6.043 & 343 & 6.043 & \\
\hline París & 21 & 6.572 & 511 & & 6.572 \\
\hline aclarisca & 21 & 6.577 & 591 & & 6.577 \\
\hline \multicolumn{4}{|c|}{ Significación } & 1 & 1 \\
\hline
\end{tabular}

En el gráfico de la figura 2 se aprecia también como esta informante realiza la sibilante del incremento -isc- próxima a la sibilante alveolar sorda del contexto Paris, mientras que la sibilante del contexto aclarix se realiza en frecuencias más bajas, es decir, más posterior que las sibilantes de los otros dos contextos.

El análisis estadístico demuestra, por lo tanto, que en esta informante no opera la analogía de la alveolopalatal final de palabra del contexto aclarix sobre la consonante sibilante del incremento -isc-, presente en el contexto aclarisca, ya que esta sibilante presenta unas características acústicas similares a las de la sibilante del contexto París. Conviene notar que, como la diferencia entre $/ \mathrm{s} / \mathrm{y} / \mathrm{g} /$ es mínima en esta variedad, incluso una pequeña presión analógica en la dirección de / $/$ / podría haber favorecido el solapamiento entre la / // y la sibilante del incremento incoativo, pero no se advierte rastro alguno de esta tendencia.

5 Como hemos indicado en la metodología, se ha examinado que no existan pausas tras las sibilantes en los contextos París y aclarix que pudieran provocar un descenso de la frecuencia del CDG de estas consonantes. Para comprobar que no existe tal efecto, además, hemos comparado la media del CDG de la sibilante de esta informante en los contextos París y aclarix con la media del CDG en los contextos rici [ri'si] 'ricino' (6706 Hz) y (e)ixir [i'fir] 'salir' ( $5257 \mathrm{~Hz}$ ), analizados en un estudio paralelo (Cruselles, en preparación). Si existiera un efecto de la frontera de palabras en los contextos París y aclarix, sus valores de CDG deberían ser menores que los de ricí y (e)ixir, respectivamente. El valor del contexto París $(6572 \mathrm{~Hz})$ es ligeramente menor que el del contexto ricí, lo que podría tener relación con la frontera entre palabras, pero la diferencia es mínima $(134 \mathrm{~Hz})$ para considerar realmente un efecto de pausa. En el caso de aclarix $(6043 \mathrm{~Hz})$, el valor es superior al del contexto (e)ixir y, por tanto, la separación estadística observada entre aclarix y aclarisca parece independiente del posible efecto de la frontera. 


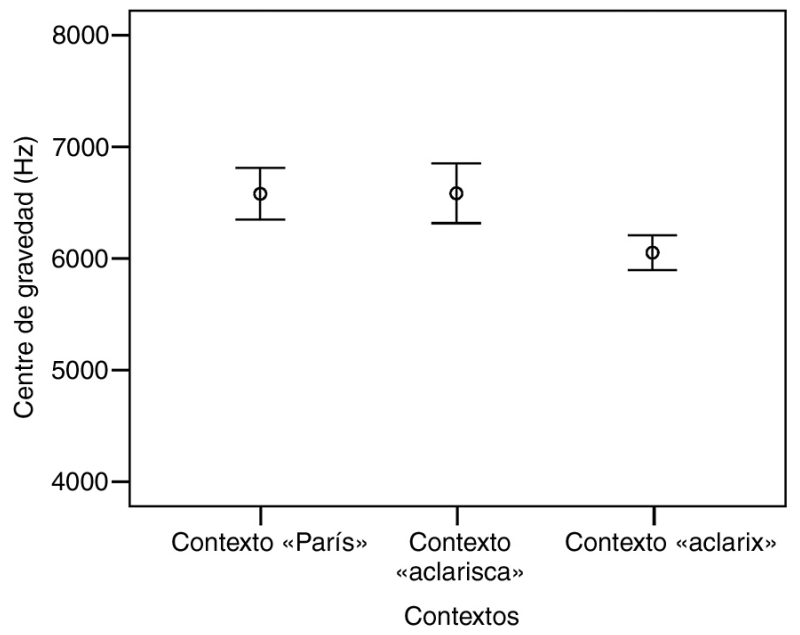

Figura 2. Gráfico de la media del CDG, con barras de error, de las sibilantes para la informante MB de Riba-roja en los contextos /-ís\#\#k-/ (Paris), /-sk-/ (aclarisca) y /-îf\#\#k-/ (aclarix).

\subsection{VARIEDAD CON DISTINCIÓN ROBUSTA}

En este apartado presentamos los resultados del test ANOVA de un factor para la informante SM de Crevillent. De acuerdo con el estudio que estamos desarrollando (Cruselles, en preparación), esta informante representa un patrón alternativo para la distinción entre / $/ \mathrm{y} / \mathrm{s} /$ en valenciano, ya que se caracteriza por una distinción robusta de estas consonantes, basada en la realización más atrasada de la sibilante alveolopalatal, una posibilidad ya apuntada en el estudio descriptivo de Beltran y Segura-Llopes (2017). La mayor posterioridad en la producción de /s/ permite distinguir claramente la sibilante alveolopalatal de la sibilante alveolar.

El análisis del centro de gravedad indica que la realización de las sibilantes en los contextos analizados es estadísticamente diferente $([\mathrm{F}(2,60)=8,129, \mathrm{p}<$ $0,001])$. Como se puede comprobar en la tabla 5 , los valores de las medias de CDG de las sibilantes en los tres contextos analizados presentan valores bastante separados entre sí: la sibilante del contexto aclarix se sitúa en frecuencias bajas, alrededor de los $4800 \mathrm{~Hz}$; la del contexto aclarisca se realiza un poco más adelantada, puesto que la media es un poco más elevada, sobre los $5800 \mathrm{~Hz}$, y la sibilante de contexto París se caracteriza por la frecuencia más alta, por encima de los $6000 \mathrm{~Hz}$. El resultado de la diferenciación entre la /s/ de París y la / $/$ / de aclarix es el esperado, porque, como se ha comprobado en estudios previos, la distinción entre estos fonemas de esta informante es muy robusta. Una vez establecido que existen diferencias estadísticas entre las sibilantes, realizamos post-hoc de Scheffé para ver las agrupaciones. Como podemos ver en la tabla 5, las sibilantes se pueden clasificar en tres grupos 
consistentemente distintos ( $\mathrm{p}=1$ ), lo que indica que los valores de CDG para cada tipo de sibilante estudiada son estadísticamente diferentes ${ }^{6}$.

TABLA 5. MEDIAS, DESVIACIÓN TÍPICA Y AGRUPACIONES (SEGÚN EL TEST POST-HOC

DE SCHEFFÉ REALIZADO A PARTIR DEL VALOR DE CENTRO DE GRAVEDAD) DE LAS

SIBILANTES EN LOS CONTEXTOS /-îf\#\#k-/, I-sk-/ Y /-ís\#\#k-/ CORRESPONDIENTE A

LOS DATOS DE LA INFORMANTE SM DE CREVILLENT

\begin{tabular}{|c|c|c|c|c|c|c|}
\hline \multirow[t]{2}{*}{ Contexto } & \multirow[t]{2}{*}{ Casos } & \multirow[t]{2}{*}{ Media } & \multirow[t]{2}{*}{ DESVIACIÓN TÍPICA } & \multicolumn{3}{|c|}{$\begin{array}{l}\text { Prueba POST-HOC De SCheffé, SUbCoN- } \\
\text { Juntos PARA ALFA }=0,05\end{array}$} \\
\hline & & & & 1 & 2 & 3 \\
\hline aclarix & 21 & 4.839 & 288 & 4.839 & & \\
\hline aclarisca & 21 & 5.876 & 305 & & 5.87 & \\
\hline París & 21 & 6.235 & 422 & & & 6.235 \\
\hline \multicolumn{4}{|c|}{ Significación } & 1 & 1 & 1 \\
\hline
\end{tabular}

En el gráfico de la figura 3 se ilustra también que la informante SM de Crevillent realiza la sibilante del incremento -isc- entre la sibilante alveolar sorda del contexto París y la sibilante alveolopalatal sorda del contexto aclarix, es decir, en un punto intermedio entre estos dos fonemas.

La triple clasificación de las sibilantes de los contextos estudiados, con la variante del contexto aclarisca situada a medio camino entre $/ \mathrm{s} / \mathrm{y} / \mathrm{s} /$, es muy indicativa para comprender la interacción entre la fonología y la morfología. Por una parte, la realización intermedia indica claramente que existe una tendencia a extender analógicamente la pronunciación alveolopalatal sorda de la terminación -ix al incremento -isc-; solo por este motivo se puede justificar la diferencia notable, de $1000 \mathrm{~Hz}$, entre el contexto París y el contexto aclarisca. Pero, por otra parte, la pronunciación recogida indica que existe otro factor, con toda probabilidad la restricción fonológica en contra de la aparición de una secuencia con una consonante palatal en la coda seguida de una consonante en el ataque, que evita que se iguale la sibilante del incremento -isc- a la consonante final del entorno aclarix.

${ }^{6}$ Como en el caso de la informante de Riba-roja, se ha comprobado que no existan pausas en los contextos París y aclarix que pudieran ocasionar un descenso de la frecuencia del CDG. Además, hemos comparado, como en el caso anterior, la media del CDG de la sibilante de esta informante en los contextos París y aclarix con la de los entornos rici $(6405 \mathrm{~Hz})$ y (e)ixir $(3761 \mathrm{~Hz})$, extraídos de un estudio paralelo (Cruselles, en preparación). Para esta informante, el valor del contexto París $(6235 \mathrm{~Hz})$ es menor que el del contexto rici, lo que podría tener alguna relación con la frontera entre palabras; con todo, incluso sumando este efecto, se mantiene la diferencia respecto del contexto aclarisca $(5876 \mathrm{~Hz})$. En el caso de aclarix $(4839 \mathrm{~Hz})$, el valor del CDG es superior al del contexto (e)ixir y, por lo tanto, como en el caso de la informante de Riba-roja, la separación estadística entre aclarix y aclarisca no parece deberse a la influencia de una posible pausa entre palabras. 


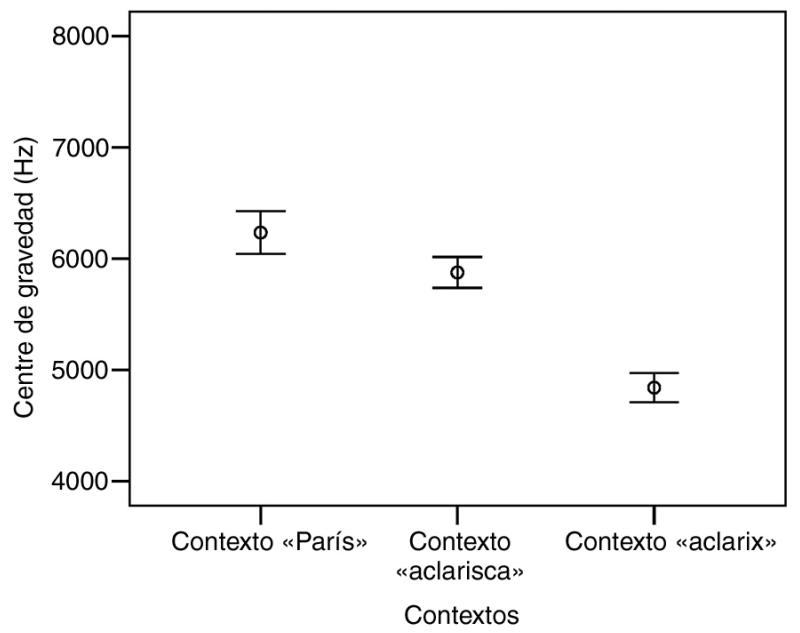

Figura 3. Gráfico de la media del CDG, con barras de error, de las sibilantes para la informante SM de Crevillent en los contextos /-ís\#\#k-/ (París), /-sk-/ (aclarisca) y /-îj\#\#k-/ (aclarix).

\section{CONCLUSIÓN}

El análisis de la pronunciación de la secuencia del incremento -isc-en valenciano, realizado a partir de tres sujetos, demuestra que existen diferentes realizaciones, motivadas tanto por condicionantes fonológicos, que favorecen la realización de la sibilante como alveolar, como morfológicos, que promueven la palatalización analógica de la sibilante. El primer sujeto ( $\mathrm{MC}$ de Albocàsser) se queda al margen de la discusión sobre la posible pronunciación palatal de la sibilante de -isc-, puesto que neutraliza la alveolopalatal sorda / $/$ / con la alveolar sorda /s/y realiza las dos consonantes siempre como una alveolar [s]. La informante de Riba-roja, por su parte, presenta una distinción mínima entre /s/ y / / y resuelve el conflicto entre fonología y morfología en favor de la restricción fonológica, ya que realiza el incremento incoativo con una sibilante alveolar sorda, a pesar de crear de esta manera una duplicidad de pronunciaciones para la sibilante en las formas incoativas: - $i s c$ - [-ísk-] vs. -ix[-íf-]. Finalmente, en la tercera hablante encontramos que es el sujeto más relevante para el estudio. Los resultados para esta informante confirman que, para algunos hablantes, la forma -ixc- no representa solo una variante ortográfica, sino que la sibilante que contiene se pronuncia, efectivamente, de manera diferente de la consonante /s/ de París. Esta realización justificaría la aparición en los textos del catalán occidental, a partir del siglo xv, de las formas incoativas como -ixc-. Con todo, la extensión de la palatalización de la forma -ix-a la forma -isc- o bien no es completa o bien se está perdiendo una vez completada, ya que la sibilante de la última secuencia no se realiza como una $[S]$, sino como una consonante a medio camino entre las sibilantes de los dos contextos de control: la [s] de París y la [J] aclarix. 
Esta realización intermedia indica que, en la gramática de la informante de Crevillent, la pugna entre la presión morfológica en favor de la igualación del paradigma y la restricción fonológica en contra de una consonante palatal en coda seguida de otra consonante dentro de la misma palabra se resuelve con una solución de compromiso. $\mathrm{O}$, dicho de otra manera, el conflicto entre la morfología y la fonología no tiene por qué resolverse con la prevalencia de uno de los dos principios, sino que también existen soluciones intermedias que muestran que las dos clases de principios pueden estar activas en la lengua a la vez.

ReCibido: mayo de 2019; ACEPTADo: octubre de 2019. 


\section{BIBLIOGRAFÍA}

Acadèmia valenciana de la llengua (AVL) (2006): Gramàtica normativa valenciana, València: Publicacions de l'Acadèmia Valenciana de la Llengua.

Beltran, Vicent y Carles Segura-Llopes (2017): Els parlars valencians, València: Universitat de València.

Boersma, Paul y David Weenink (2014): Praat: doing phonetics by computer, vers. 5.0.02. Programari disponible en línea a: <http://www.praat.org/>.

Casanova, Emili (1993): «El català dins la Romània: a propòsit del doblet ESC/ISC», en Ramón Lorenzo Vázquez (coord.), Actas do XIX Congreso Internacional de Lingüistica e Filoloxía Románicas, A Coruña: Fundación Pedro Barrié de la Maza, Conde Fenosa, 293-315.

Colon, Germà (1970): «Sobre el funcionament de les sibilants en el català de Castelló», en Phonétique et Linguistique Romanes, Mélanges offerts à M. Georges Straka, vol. 1., Lyon / Strasbourg, Société de linguistique romane, 43-51.

Cruselles, Paula, Ricard Herrero y Jesús Jiménez (2017): «Propietats acústiques i percepció de contrastos mínims en les sibilants del valencià», Estudios de Fonética Experimental XXVI: 93-132.

Cruselles, Paula (en preparación): Estudi acústic de les fricatives del valencià», tesis doctoral, Universitat de València.

IBM Corp. Released 2016. IBM SPSS Statistics for Windows, Version 24.0. Armonk, Nueva York: IBM Corp.

Jongman, Allard, Ratree Wayland y Serena Wong (2000): «Acoustic characteristics of English fricatives", Journal of the Acoustic Society of America 108 (3): 1252-1263.

Lloret, Maria-Rosa (2008): «Estructura sil-làbica», en Gramàtica del català contemporani, vol. 1., Barcelona, Empúries, 195-249.

Pérez Saldanya, Manuel (1998): Del llati al català. Morfosintaxi verbal històrica, València: Universitat de València.

Recasens, Daniel (1986): Estudis de fonètica experimental del català oriental central, Barcelona, Publicacions de l'Abadia de Montserrat.

Recasens, Daniel (1993): Fonètica i fonologia, Barcelona: Enciclopèdia Catalana.

Recasens, Daniel (1996): Fonètica descriptiva del català: Assaig de caracterització de la pronúnica del vocalisme i consonantisme del català al segle $X X$, Barcelona: Institut d'Estudis Catalans.

ReCasens, Daniel (2014): Fonètica i fonologia experimental del català: vocals i consonants, Barcelona: Institut d'Estudis Catalans.

Recasens, Daniel y Aina Espinosa (2006): «Estudi experimental de les consonants fricatives del mallorquí i del valencià», Estudis Romànics 28: 125-150.

Recasens, Daniel y Aina Espinosa (2007): «An electropalatographic and acoustic study of affricates and fricatives in two Catalan dialects", Journal of the International Phonetic Association 37.2: 143-172.

SABorit, Josep (2009): Millorem la pronúncia, València: Acadèmia Valenciana de la Llengua.

Sanchis Guarner, Manuel (1950): Gramàtica Valenciana, València: Torre.

SARAGOssà, Abelard (2005): Gramàtica valenciana raonada i popular: els fonaments, València: Tabarca. 
Segura-Llopes, Carles (2003): Una cruillla lingüistica. Caracterització del parlar del Baix Vinalopó, Alacant: Universitat d'Alacant-Departament de Filologia Catalana.

WheELER, Max (1987): «L'estructura fonològica de la sílllaba i del mot en català», Estudis de Llengua i Literatura Catalanes (Miscel.lània Antoni M. Badia i Margarit) XIV: 79-108.

WheEler, Max (1993): «On the hierarchy of naturalness principles in inflexional morphology», Journal of Linguistics 29: 55-111. 